\title{
COMPARAÇÃO DA MODULAÇÃO AUTONÔMICA CARDÍACA DURANTE ESFORÇO DE FUMANTES E NÃO FUMANTES
}

\author{
COMPARISON OF CARDIAC AUTONOMIC MODULATION DURING EFFORT OF SMOKERS \\ AND NON-SMOKERS

\section{COMPARACIÓN DE LA MODULACIÓN AUTONÓMICA CARDÍACA DURANTE ESFUERZO DE LOS FUMADORES Y LOS NO FUMADORES}

\author{
Larissa Aimeê Assunção Alves \\ (Fisioterapeuta) \\ Juliana Braga de Oliveira \\ (Fisioterapeuta) \\ Rafael Leite Alves² (Fisioterapeuta) \\ Pedro Henrique Scheidt Figueiredo \\ (Fisioterapeuta) \\ Marco Fabrício Dias Peixoto \\ (Educador Físico) \\ Márcia Maria Oliveira Lima \\ (Fisioterapeuta)

\section{Universidade Federal dos Vales do Jequitinhonha e Mucuri (UFVJM), Diamantina, MG, Brasil. \\ 2. Universidade Federal de Minas Gerais (UFMG), Faculdade de Medicina, Belo Horizonte, MG, Brasil.}

\section{Correspondência:}

Márcia Maria Oliveira Lima Universidade Federal dos Vales do Jequitinhonha e Mucuri. Departamento de Fisioterapia. Laboratório de Reabilitação Cardiovascular. Rod.: MGT 367 - Km 583, no 5000. Alto da Jacuba. Diamantina, MG, Brasil. 39100-000. marcialima_ufvjm@yahoo.com.br

\section{RESUMO}

Introdução: O tabagismo é uma das principais causas de morbimortalidade em todo o mundo. Indivíduos fumantes têm risco aumentado de desenvolver disfunção autonômica, a qual pode ser avaliada tanto pela variabilidade da frequência cardíaca (VFC) como pela frequência cardíaca de recuperação (FCRec). A maioria dos estudos envolvendo esta análise é realizada em adultos de idade avançada e na condição de repouso. Objetivos: Comparar a atividade autonômica cardíaca em repouso e esforço, em homens jovens, fumantes e não fumantes. Métodos: Trinta e dois jovens voluntários, (idade 22,0 2 2,8 anos) foram distribuídos em dois grupos: o grupo fumante (GF; $n=15$ ) e o não fumante (GNF; $n=17)$. Realizou-se o teste de Cooper, com análise da VFC pelo cardiofrequencímetro Polar ${ }^{\circledR}$ S810i, em repouso e durante o esforço e FCRec. Resultados: No GF, 73\% foram classificados com nível de dependência nicotínica "muito baixa" segundo questionário de Fargeström. A classificação de ativos e muito ativos pelo questionário IPAQ correspondeu a mais de $50 \%$ da amostra em ambos os grupos. Não se observou diferenças significativas entre os grupos na VFC, tanto no repouso quanto no esforço. Entretanto, em cada grupo, notou-se diferença na maioria dos índices de VFC do repouso para o esforço. No teste de Cooper não foram observadas diferenças significativas na FCmédia, FCpico e de FCRec entre os grupos, mas notou-se uma melhor capacidade funcional no GNF pela distância caminhada (2050,2 $\pm 300,0$ vs. 1780,3 $\pm 390,4 \mathrm{~m}, \mathrm{p}=0,036)$. Conclusão: O GF apresentou menor capacidade funcional e ativação parassimpática durante o esforço, além de menores índices de VFC durante repouso, o que pode sugerir um comprometimento precoce na modulação autonômica cardíaca.

Palavras-chave: exercício, hábito de fumar, disautonomias primárias.

\section{ABSTRACT}

Introduction: Smoking is one of the major causes of morbidity and mortality worldwide. Smokers have an increased risk of developing autonomic dysfunction, which can be evaluated by both heart rate variability (HRV) and heart rate recovery (HRRec). Most studies involving this analysis is performed on adults of advanced age and in condition of rest. Objectives: To compare the cardiac autonomic activity, at rest and during exercise in young men, smokers and non-smokers. Methods: Thirty-two young volunteers (aged $22.0 \pm 2.8$ years) were divided into two groups: the smoking group (SG, $n=15$ ) and non-smokers (NSG, $n=17$ ). We carried out the Cooper test and HRV analysis by heart rate monitor Polar ${ }^{\circledR}$ S810i, at rest and during exercise and HRRec. Results: In SG, 73\% were classified as having a "very low" level of nicotine dependence according to the Fargeström's questionnaire. Classification of active and very active by the IPAQ questionnaire accounted for over $50 \%$ of sample in both groups. There were no significant differences between groups in HRV, both at rest and during exercise. However, in each group, difference has been noted in most HRV indexes from rest to exercise. During Cooper test there were no significant differences in mean HR, HRpeak and HRRec between groups, but a better functional capacity was noted in NSG in the distance walked (2050.2 \pm 300.0 vs. $1780.3 \pm 390.4 \mathrm{~m}, p=0.036$ ). Conclusion: The SG showed lower functional capacity and parasympathetic activation during exercise, besides lower HRV indexes during rest, suggesting an early impairment in cardiac autonomic modulation.

Keywords: exercise, smoking, primary dysautonomias.

\section{RESUMEN}

Introducción: El consumo de cigarrillos es una de las más importantes causas de morbilidad y mortalidad en todo el mundo. Los individuos fumadores tienen mayor riesgo de desarrollar disfunción autonómica, que puede ser evaluada tanto por la variabilidad del ritmo cardíaco (VRC) como por la frecuencia cardiaca de recuperación (FCrec). La mayoría de los estudios con estos análisis se lleva a cabo en las personas más viejas y en situación de reposo. Objetivos: Comparar la actividad autonómica cardiaca, en momento de reposo y durante ejercicios, en hombres jóvenes, fumadores y no fumadores. Métodos: Treinta y dos voluntarios jóvenes (edad 22,0 $\pm 2,8$ años) 
fueron divididos en dos grupos: grupo de fumadores (GF; $n=15)$ y no fumadores (GNF; $n=17)$. Se realizó la prueba de Cooper con el análisis de la VRC por pulsómetro Polar ${ }^{\circledR}$ S810i, durante reposo y ejercicio y FCrec. Resultados: En el GF, el 73\% fueron clasificados con niveles de dependencia por la nicotina "muy bajo", según cuestionario Fargeström. La clasificación de activos y muy activos, mediante cuestionario IPAQ, correspondió a más de 50\% de la amuestra en ambos los grupos. No hubo diferencia significativa entre los grupos en la VRC, tanto en reposo cuanto en ejercicio. Sin embargo, en cada grupo, observamos una diferencia en la mayoría de los índices de VRC del reposo para el ejercicio. En la prueba de Cooper no se observó diferencias significativas en las FC media, FCpico y FCrec, pero se verificó una mejor capacidad funcional en el GNF por la distancia caminada (2050.2 \pm 300.0 vs. 1780,3 $\pm 390,4 \mathrm{~m}$, p =0,036). Conclusión: El GF mostró una capacidad funcional y una activación parasimpática más baja durante el ejercicio, asi como tasas más bajas de VRC en reposo, lo que puede sugerir un deterioro temprano en la modulación autonómica cardíaca.

Palabras clave: ejercicio, hábito de fumar, disautonomías primarias.

\section{INTRODUÇÃO}

O tabagismo é uma das principais causas de mortalidade e morbidade em todo o mundo. É considerado o principal fator de risco para diversas doenças, dentre as quais câncer de pulmão, doença arterial coronariana, infarto agudo do miocárdio e doenças respiratórias ${ }^{1,2}$. Estima-se que esse hábito seja a principal causa de morte evitável no mundo ${ }^{3}$, e que quanto maior a exposição ao tabaco, maiores são as morbidades associadas 4 .

Em relação ao sistema cardiovascular, indivíduos fumantes têm risco aumentado de deterioração das propriedades elásticas da artéria aorta, com aumento da pressão arterial (PA), prejuízo da função ventricular, inadequação na remodelação cardíaca, além de disfunção autonômica e endotelial ${ }^{5,6}$.

A disfunção do sistema nervoso autonômico (SNA) é caracterizada basicamente por um aumento da ativação simpática e redução do tônus vagal de repouso ${ }^{7}$. A avaliação da função autonômica cardíaca pode ser realizada pela análise da variabilidade da frequência cardíaca (VFC) e da medida da frequência cardíaca de recuperação (FCrec) ${ }^{4}$.

Uma menor VFC durante o esforço está associada com maior morbidade e mortalidade cardiovascular, devido a uma maior ativação do ramo simpático do SNA ${ }^{3}$, assim como uma menor FCrec é também um preditor independente de mortalidade entre adultos saudáveis ${ }^{8}$. Tanto a VFC como a FCrec têm sido utilizadas para avaliar a disfunção autonômica em fumantes ${ }^{4,9}$, no entanto, a maioria dos estudos é realizada em adultos de idade avançada e na condição de repouso apenas ${ }^{10,11}$.

Dessa forma, esse estudo comparou a atividade autonômica cardíaca ao repouso e esforço, em homens jovens fumantes e não fumantes.

\section{MÉTODO}

Este estudo foi parte de uma pesquisa que objetivou avaliar o nível de atividade física e qualidade de vida de jovens universitários, onde se avaliou um total de 189 indivíduos. Considerados os critérios de inclusão e exclusão deste estudo, a amostra resultou em um número final de 32 indivíduos distribuídos em um grupo fumante (GF; $n=15$ ) e outro grupo não fumante (GNF; $n=17)$. Esta pesquisa foi aprovada pelo Comitê de Ética em Pesquisa da Universidade Federal dos Vales do Jequitinhonha e Mucuri (UFVJM), parecer n 137/10, e os participantes assinaram voluntariamente o Termo de Consentimento Livre e Esclarecido.

Para serem incluídos no sub-estudo os voluntários deveriam ser alunos regularmente matriculados na UFVJM; serem do gênero masculino; terem idade entre 18 e 30 anos; e não apresentarem distúrbios musculoesqueléticos, respiratórios, cardiovasculares, metabólicos e neurológicos que contra indicassem a realização do teste de Cooper.
Para os fumantes, era também necessário que apresentassem a mínima dependência nicotínica de acordo com o questionário de Fargeström, fumar por mais de três anos e consumir no mínimo três cigarros de palha ou seis cigarros brancos por dia (um cigarro de palha $=$ dois cigarros de papel) $)^{12}$. Este instrumento consiste de seis perguntas com alternativas pontuadas. O grau de dependência é dado pela soma dos pontos, a qual os classifica em: muito baixa, baixa, média, elevada e muito elevada ${ }^{13}$.

Foram excluídos aqueles em uso de medicamento para controle do tabagismo e/ou com ação cronotrópica e obesos.

Os indivíduos foram orientados a não fazer uso de álcool e cafeína por pelo menos 12 horas antes das sessões de avaliação; não realizarem exercício físico e ou atividade física vigorosa e incluir refeições leves e uma noite de sono adequada nas 24 horas anteriores à avaliação; abster-se de alimentar por quatro horas e de fazer uso de cigarro por pelo menos 30 minutos antes da avaliação ${ }^{14}$.

Foi utilizada uma ficha de avaliação contendo, além da identificação, dados como pressão arterial (PA), FC, peso, altura, índice de massa corporal (IMC) e nível de aptidão física pelo IPAQ versão $8^{15}$. A PA foi aferida pelo método auscultatório, seguindo as VI Diretrizes Brasileiras de Hipertensão Arterial ${ }^{16}$. Obtiveram-se as medidas de massa corporal e estatura por meio de uma balança devidamente calibrada (LD 1050, Líder $^{\circledR}$, Brasil) com estadiômetro embutido, com posterior cálculo do IMC, o qual foi calculado pela razão: peso/altura².

Para análise da capacidade funcional utilizou-se o Teste de Cooper (TC) ${ }^{14}$. Antes do teste os voluntários permaneceram 10 minutos sentados para estabilização hemodinâmica, onde foram avaliadas a PA, FC e freqüência respiratória (FR). Os indivíduos foram orientados a interromper o teste caso apresentassem sintomas tais como: câimbras, tonteiras, náuseas, dores, dispnéia ou qualquer outra percepção subjetiva que o impedisse de concluí-lo. Durante o teste, foram orientados a andar ou correr em sua capacidade máxima, durante 12 minutos, em pista plana de 40 metros. Após o teste, o valor da distância percorrida foi utilizado como marcador da capacidade funcional ${ }^{14}$.

A análise da VFC foi realizada por cardiofrequencimetro (Polar ${ }^{\circledR}$ S810i; Finlândia) ${ }^{17}$. Os dados foram registrados em duas fases: o repouso pré-exercício (1a fase) com duração de 10 minutos, sendo analisados os cinco minutos finais; e durante esforço no TC ( $2^{\text {a }}$ fase) a qual teve duração de 12 minutos. A FR foi controlada, sendo mantida em torno de 15 incursões respiratórias por minuto (irpm) para inicio de registro de iR-R. O estudo da VFC foi realizado pelo método linear, através dos domínios do tempo e da freqüência. O índice considerado no domínio do tempo foi o rMSSD (raiz quadrada da média do quadrado das diferenças entre intervalos RR normais adjacentes). Já no domínio da 
frequência utilizaram-se os componentes de alta frequência (AF) e o de baixa frequência (BF), e também a razão entre esses índices, BF/ $A F$, que reflete o balanço simpato-vagal ${ }^{18}$.

O valor da FCrec foi calculado pela diferença entre a FC no final do teste de Cooper a FC no primeiro minuto pós-esforço durante a recuperação ativa.

\section{Análise Estatística}

O cálculo amostral foi realizado tendo como variável de desfecho as alterações esperadas na variável rMSSD. Foi calculado o número de 16 indivíduos em cada grupo, considerando uma diferença de 35,2\%, desvio padrão de $33 \%$, alfa de $5 \%$ e poder do teste de $80 \%{ }^{7}$. Utilizou-se o pacote estatístico Statistical Package for the Social Sciences (SPSS 17.0, USA). Considerou-se como nível de significância valor menor que 0,05. O teste de Kolmogorov-Smirnov foi usado para avaliar normalidade dos dados, os quais são apresentados em média e desvio padrão (normais) ou mediana e intervalo interquartílico (não normais). Na analise entre os grupos aplicou-se o teste $t$ não pareado ou Mann-Whitney teste, e na análise intra-grupo teste t pareado ou Wilcoxon, conforme apropriado. A comparação do nível de aptidão física pelo IPAQ entre os grupos foi realizada pelo teste qui-quadrado.

\section{RESULTADOS}

Considerando-se o questionário de Fargeström, 73\% dos indivíduos do GF, foram considerados com nível de dependência nicotínica "muito baixa". Em relação ao IPAQ, em ambos os grupos, a classificação de ativos e muito ativos correspondeu a mais de $50 \%$ da amostra (tabela 1).

A tabela 1 apresenta as características dos participantes do estudo. Entretanto, apesar de não existir diferenças significativas em relação aos valores do IMC, observa-se que os grupos diferem em relação à classificação, onde o GF encontra-se na faixa de sobrepeso e o GNF na faixa de normalidade ${ }^{14}$. Houve diferença significativa em relação á PAD entre os dois grupos, porém sem significância clínica. Em relação ao comportamento da VFC durante repouso observa-se na tabela 2

Tabela 1. Caracterização da amostra estudada.

\begin{tabular}{c|c|c|c}
\hline Variável & Grupo fumante $(\mathbf{n = 1 7 )}$ & Grupo não fumante $(\mathbf{n = 1 8 )}$ & $\boldsymbol{P}$ \\
\hline Idade $(\mathrm{anos})$ & $21,5 \pm 1,9$ & $22,5 \pm 3,4$ & 0,339 \\
\hline IMC $\left(\mathrm{Kg} / \mathrm{m}^{2}\right)$ & $25,9 \pm 4,9$ & $23,9 \pm 3,8$ & 0,195 \\
\hline PAS $(\mathrm{mmHg})$ & $118,9 \pm 7,7$ & $116,7 \pm 11,9$ & 0,539 \\
\hline PAD $(\mathrm{mmHg})$ & $70,0(70,0$ a 70,0$)$ & $80,0(69,0$ a 83,0$)$ & 0,018 \\
\hline FC $(\mathrm{bpm})$ & $88,1 \pm 4,9$ & $87,8 \pm 3,5$ & 0,959 \\
\hline IPAQ $(\mathrm{n})$ & $6 / 4 / 3 / 1 / 1$ & $4 / 8 / 2 / 3 / 0$ & 0,430 \\
\hline
\end{tabular}

Dados apresentados por média \pm desvio padrão ou mediana (intervalo interquartílico); IMC: índice de massa corporal; PAS: pressão arterial sistólica; PAD: pressão arterial diastólica; FC: freqüência cardíaca; IPAQ - muito ativo/ ativo/ irregularmente ativo $\mathrm{A}$ / irregularmente ativo $\mathrm{B}$ / sedentário.

Tabela 2. Comparação dos índices da VFC entre os grupos fumante e não fumante no repouso e durante o esforço.

\begin{tabular}{|c|c|c|c|c|}
\hline \multirow[t]{2}{*}{ Variável } & \multicolumn{2}{|c|}{ Fumante } & \multicolumn{2}{|c|}{ Não fumante } \\
\hline & Repouso & Esforço & Repouso & Esforço \\
\hline $\mathrm{rMSSD}(\mathrm{ms})$ & $\begin{array}{c}79,1 \\
(43,4 \text { a } 123,9)\end{array}$ & $\begin{array}{c}28,9^{*} \\
(4,2 \text { a } 102,9)\end{array}$ & $\begin{array}{c}107,3 \\
(39,5 \text { a } 187,0)\end{array}$ & $\begin{array}{c}125,7 \\
(11,4 \text { a } 194,8)\end{array}$ \\
\hline$B F\left(m s^{2}\right)$ & $\begin{array}{c}3504,6 \\
(1702,0 \text { a } 8811,1)\end{array}$ & $\begin{array}{c}257,2^{*} \\
(21,1 \text { a } 486,0)\end{array}$ & $\begin{array}{c}3466,5 \\
(2432,7 \text { a } 8312,2)\end{array}$ & $\begin{array}{c}827,0^{*} \\
(95,1 \text { a } 2188,3)\end{array}$ \\
\hline$A F\left(m s^{2}\right)$ & $\begin{array}{c}1923,4 \\
(494,1 \text { a } 5328,1)\end{array}$ & $\begin{array}{c}81,4^{*} \\
(4,1 \text { a } 1044,4)\end{array}$ & $\begin{array}{c}2378,8 \\
(600,6 \text { a } 9039,3)\end{array}$ & $\begin{array}{c}2274,1^{*} \\
(26,4 \text { a } 3870,8)\end{array}$ \\
\hline $\mathrm{BF} / \mathrm{AF}(\%)$ & $\begin{array}{c}159,1 \\
(106,7 \text { a } 294,2)\end{array}$ & $\begin{array}{c}117,1 \\
(61,3 \text { a } 612,6)\end{array}$ & $\begin{array}{c}151,7 \\
(79,1 \text { a } 509,0)\end{array}$ & $\begin{array}{c}57,5 \\
(46,6 \text { a } 447,7)\end{array}$ \\
\hline
\end{tabular}

Dados apresentados pela mediana (intervalo interquartílico); rMSSD= corresponde raiz quadrada da média do quadrado das diferenças entre intervalos $\mathrm{RR}$ normais adjacentes; $\mathrm{BF}=$ baixa frequência; $\mathrm{AF}=$ alta frequência; $\mathrm{BF}$ / $A F=$ razão da baixa frequência pela alta frequência; * $p<0,05$ em relação ao repouso. que não houve diferenças entre os grupos para nenhuma das variáveis analisadas (poder do teste para a variável rMSSD = 83\%). A figura 1 ilustra as variações na VFC intragrupo, do momento de repouso para o de esforço, em ambos os grupos. Observa-se uma queda significativa para maior parte dos índices nos dois grupos, com exceção do rMSSD no GNF e da relação BF/AF em ambos os grupos. Entretanto, na comparação dos valores do $\triangle$ entre os grupos, como demonstradas na tabela 3, as diferenças não foram significativas.

Na tabela 3 observa-se o comportamento dos dois grupos durante o teste de Cooper. Nota-se uma melhor capacidade funcional pela maior distância percorrida no GNF. Nenhuma diferença significativa foi observada na FCpico, FCmédia e FCrec.



Figura 1. Avaliação intragrupo do comportamento dos índices de VFC entre os períodos de repouso e esforço.

Tabela 3. Comparação das variáveis cardiovasculares analisadas durante o Teste de Cooper e da diferença $(\Delta)$ entre esforço e repouso nos índices da VFC entre os grupos fumante e não fumante.

\begin{tabular}{|c|c|c|c|}
\hline Variável & Grupo fumante & Grupo não fumante & $P$ \\
\hline Distância (m) & $1780,3 \pm 390,4$ & $2050,2 \pm 300,0$ & 0,036 \\
\hline FCpico (bpm) & $203(185,0$ a 216,0$)$ & $214,0(196,5$ a 237,5$)$ & 0,199 \\
\hline FCmédia (bpm) & $154,4 \pm 27$ & $160,6 \pm 18,9$ & 0,455 \\
\hline FCrec (bpm) & $30,1 \pm 15,0$ & $33,0 \pm 10,3$ & 0,519 \\
\hline$\Delta \mathrm{rMSSD}(\mathrm{ms})$ & $-57,4(-119,7$ a 10,6$)$ & $-24,1(-53,6$ a 29,7$)$ & 0,234 \\
\hline$\Delta \mathrm{BF}\left(\mathrm{ms}^{2}\right)$ & $-2716(-8553,9$ a $-1039,7)$ & $-2823,9(-7315,8$ a $-1596,1)$ & 0,637 \\
\hline$\triangle A F\left(m s^{2}\right)$ & $-1240,5(-5323,9$ a $-356,5)$ & $-752,1(-6044,2$ a $-306,7)$ & 0,835 \\
\hline$\triangle \mathrm{BF} / \mathrm{AF}(\%)$ & $-12,4(-69,5$ a 418,0$)$ & $-39,4(-81,9$ a 12,6$)$ & 0,396 \\
\hline
\end{tabular}

Dados apresentados por média \pm desvio padrão ou mediana (intervalo interquartílico)rMSSD= corresponde raiz quadrada da média do quadrado das diferenças entre intervalos RR normais adjacentes; $\mathrm{BF}=$ baixa frequência; $\mathrm{AF}=$ alta frequência; $\mathrm{BF} / \mathrm{AF}=$ razão da baixa frequência pela alta frequência; $m s=$ milissegundos; $F C$ : frequêencia cardíaca; FCrec: frequência cardíaca de recuperação. 


\section{DISCUSSÃO}

O propósito deste estudo em comparar dois grupos de homens jovens, um grupo fumante e outro não fumante, quanto ao comportamento da atividade autonômica cardíaca ao repouso e esforço, mostrou não haver diferença significativa entre os grupos nos dois momentos. Entretanto, do repouso para o esforço, demonstrou uma queda significativa nos índices do domínio da frequência, em ambos os grupos, mas em relação ao domínio do tempo esta redução só foi observada no GF. Adicionalmente notou-se uma melhor capacidade funcional no GNF.

No mais, apesar de ambos os grupos desenvolverem uma intensidade de esforço semelhante, como demonstrado pelas FCmédia e FCpico durante o teste de Cooper, o GNF teve melhor desempenho, representado pela maior distancia caminhada.

De um modo geral o balanço autonômico cardíaco do repouso para o exercício é caracterizado por uma diminuição imediata do tônus vagal no início do esforço, o que contribui inteiramente com o aumento inicial da FC, sendo acompanhado por uma retirada gradual desse tônus. Com o aumento do tempo e/ou da intensidade do exercício (FC em torno de 100 bpm) um efeito crescente da ativação simpática passa a ser o mecanismo principal pelo aumento da $\mathrm{FC}^{19}$. Entretanto, a intensidade de esforço na qual há o desaparecimento da influência vagal cardíaca é mais alta naqueles indivíduos que realizam atividades aeróbias habitualmente ${ }^{20}$. E mais, apesar da retirada vagal ser mais rápida em indivíduos ativos, ela ocorre em menor magnitude, fazendo com que, durante exercício, a retirada vagal seja menor no individuo mais condicionado ${ }^{19}$. Tais mecanismos podem explicar o comportamento observado neste estudo, visto a queda observada do índice rMSSD no GF durante o (figura 1), o qual apresentou um pior condicionamento cardíaco (menor distância caminhada). Além disso, indivíduos melhor condicionados apresentam menor intensificação simpática durante o exercício ${ }^{21}$.

Apesar da semelhança entre os grupos em relação ao nível de atividade física avaliado pelo IPAQ, no presente estudo notou-se uma maior capacidade funcional e um melhor comportamento autonômico frente ao estimulo no GNF, podendo sugerir que as alterações demonstradas no GF podem ser em parte justificadas pelo uso de tabaco.

A maioria dos efeitos agudos e crônicos do fumo sobre a regulação neurocardiovascular pode ser explicada pela ação da nicotina ${ }^{22}$, a qual envolve a estimulação e bloqueio dos gânglios autonômicos; a liberação de catecolaminas medulares; estimulação de adrenalina, quimiorreceptores do corpo carotídeo e barorreceptores aórticos; além da ação direta no sistema nervoso central ${ }^{23,24}$.

Outros estudos já demonstraram que o tabagismo crônico leva a ativação simpática e redução da modulação vagal, os quais se caracterizam pela diminuição dos índices de VFC em fumantes em repouso ${ }^{25,26}$ e durante exercício ${ }^{27}$. Alyan et al. ${ }^{28}$ avaliaram os efeitos do tabaco na VFC entre adultos jovens (cerca de 35 anos), fumantes e não fumantes de ambos os sexos, e observaram uma redução nos índices rMSSD e AF.

De maneira geral, no presente estudo os efeitos do tabaco podem não ter sido muito pronunciados devido a amostra ter sido classificada por baixa dependência nicotínica. Em seu estudo, Kupari et al. ${ }^{29}$ avaliaram o efeito do número de cigarros sobre índices da VFC, observando redução da mesma em indivíduos que fumavam 10 cigarros ou mais por dia quando comparados a não-fumantes ou àqueles que fumavam menos de 10 cigarros por dia.
Em relação ao IMC, foi observado que, embora os grupos não apresentassem diferença estatisticamente significante entre seus valores, apresentaram diferença quanto a sua classificação. Entretanto, acreditamos que isso não tenhar interferido em nossos resultados, pois ambos os grupos realizaram o teste em uma mesma intensidade de esforço (FCmédia e FCpico) e a relação da liberação das citocinas durante o processo inflamatório e a influência dessas na modulação autonômica está mais associada á obesidade e não ao sobrepeso ${ }^{30,31}$. A diferença estatisticamente significatica da PAD parece não interfirir na homogeneidade do grupo uma vez que está relacionada ao calibre do vaso e á resistência periférica total. Como nossa amostra de fumantes foi classificada como tendo muito baixo nível de dependência nicotínica, acreditamos que os efeitos da nicotina não tenham sido suficientes para causarem alterações deste nível. Além disso, a diferença observada não representa significância clínica.

Diferentes respostas entre fumantes e não fumantes também podem ser observadas na capacidade funcional como demonstrado por Correa e Tumelero ${ }^{32}$, que encontraram menor distância percorrida no Teste de Cooper em nove indivíduos fumantes quando comparado a nove não fumantes ativos e sedentários (idades entre 18 e 42 anos). Ao contrário, Kobayashi et al. ${ }^{9}$, avaliando 18 adultos jovens (idade média 33 anos), fumantes e não fumantes, não observou diferenças significativas no $\mathrm{VO}_{2}$ durante teste de esforço máximo em esteira. Diferenças metodológicas próprias dos dois testes podem explicar tais resultados.

No momento pós-exercicio, não se observou, nesta investigação, diferença na FCrec entre os grupos. Já Kobayashi et al. ${ }^{9}$, constataram um retardo significativo na FCrec no grupo de fumantes e relacionaram este fato com o aumento da atividade simpática e liberação de catecolaminas promovido pela nicotina absorvida pelo organismo. Entretanto, no referido estudo a FCrec foi avaliada após 3 min de recuperação, diferente do presente estudo onde se analisou a FCrec durante recuperação ativa após 1 min.

Estudos que têm por objetivo a avaliação da modulação autonômica cardíaca freqüentemente utilizam o Holter de 24 horas, entretanto o instrumento utilizado em nosso estudo foi o cardiofrequencímetro Polar (modelo S810i) já validado para este fim ${ }^{17}$. Tendo em vista que nesta amostra a dependência nicotínica foi classificada como "muito baixa", novos estudos envolvendo indivíduos com níveis mais expressivos de dependência nicotínica necessitam ser considerados. Adicionalmente, para uma análise mais específica da capacidade cardiopulmonar, estudos futuros podem verificar a possibilidade de investigar essa condição por meio do teste de esforço cardiopulmonar.

\section{CONCLUSÃO}

O grupo fumante, mesmo com nível de dependência nicotínica "muito baixa", apresentou menor capacidade funcional quando comparado ao grupo não fumante o que sugere o impacto do tabagismo no condicionamento físico. Associado a isto, o comportamento da modulação autonômica durante o exercício dos fumantes sugere uma alteração precoce na modulação autonômica cardíaca.

$\overline{\text { Todos os autores declararam não haver qualquer potencial conflito }}$ de interesses referente a este artigo. 


\section{REFERÊNCIAS}

1. Patel K, Schlundt D, Larson C, Wang H, Brown A, Hargreaves M. Chronic illness and smoking cessation. Nicotine Tob Res. 2009;11(8):933-9.

2. Zhang $M, A n Q$, Yeh F, Zhang $Y$, Howard BV, Lee ET, Zhao J. Smoking-attributable mortality in American Indians: findings from the Strong Heart Study. Eur J Epidemiol. 2015 Jul;30(7):553-61.

3. World Health Organization (WHO). Building blocks for tobacco control. Geneva: WHO;2004 Disponível:http://www.who.int/tobacco/resources/publications/tobaccocontrol_handbook/en/. Acesso em: Setembro de 2012.

4. Cagirci G, Cay S, Karakurt O, Eryasar N, Kaya V, Canga A, et al. Influence of heavy cigarette smoking on heart rate variability and heart rate turbulence parameters. Ann Noninvasive Electrocardiol. 2009;14(4):327-32.

5. Adamopoulos D, Argacha JF, Gujic M, Preumont N, Degaute JP, van de Borne P. Acute effects of nicotine on arterial stiffness and wave reflection in healthy young non-smokers. Clin Exp Pharmaco Physiol. 2009;36(8):784-9.

6. Castardeli E, Paiva SA, Matsubara BB, Matsubara LS, Minicucci MF, Azevedo PS, et al. Chronic cigarette smoke exposure results in cardiac remodeling and impaired ventricular function in rats. Arq Bras Cardiol. 2005;84(4):320-4

7. Barutcu I, Esen AM, Kaya D, Turkmen M, Karakaya O, Melek M, et al. Cigarette smoking and heart rate variability: dynamic influence of parasympathetic and sympathetic maneuvers. Ann Noninvasive Electrocardiol. 2005;10(3):324-9.

8. Cole CR, Foody JM, Blackstone EH, Lauer MS. Heart rate recovery after submaximal exercise testing as a predictor of mortality in a cardiovascularly healthy cohort. Ann Intern Med. 2000;132(7):552-5.

9. Kobayashi Y, Takeuchi T, Hosoi T, Loeppky JA. Effects of habitual smoking on cardiorespiratory responses to sub-maximal exercise. J Physiol Anthropol Appl Human Sci. 2004;23(5):163-9.

10. Manzano BM, Vanderlei LCM, Ramos EMC, Ramos D. Implicações do tabagismo sobre o controle autônomo cardíaco. Arq Ciênc Saúde 2010;17(2):97-101.

11. Arosio E, De Marchi S, Rigoni A, Prior M, Lechi A. Effects of smoking on cardiopulmonary baroreceptor activation and peripheral vascular resistance. Eur J Clin Invest. 2006:36(5):320-5.

12. de Lolio CA, de Souza JM, Santo AH, Buchalla CM. Smoking prevalence in the urban locality of southeastern Brazil. Rev Saude Publica. 1993;27(4):262-5.

13. Pietrobon RC, Barbisan JN, Manfroi WC. Utilização do Teste de dependência à nicotina de Fagerström como um instrumento de medida do grau de dependência. Rev HCPA. 2007;27(3):31-6. 8.

14. American College of Sports Medicine. Testes de esforço. In: ACSM. Diretrizes do ACSM para os testes de esforço e sua prescrição. 7a edição. Rio de Janeiro: Guanabara Koogan; 2007. p. 31-42.

15. Matsudo S, Araújo T, Matsudo V, Andrade D, Andrade E, Oliveira C, et al. Questionário Internacional de Atividade Física (IPAQ): estudo de validade e reprodutibilidade no Brasil. Rev Bras Ativ Fís Saúde. 2001;6(2):5-12.

16. Sociedade Brasileira de Cardiologoa. VI Diretriz Brasileira de Hipertensão arterial. Arq Bras Cardiol 2010; 95(Suppl 1):1-51

17. Vanderlei LC, Silva RA, Pastre CM, Azevedo FM, Godoy MF. Comparison of the Polar S810i monitor and the ECG for the analysis of heart rate variability in the time and frequency domains. Braz J Med Biol Res. 2008;41(10):854-9.

18. Heart rate variability: standards of measurement, physiological interpretation and clinical use. Task Force of the European Society of Cardiology and the North American Society of Pacing and Electrophysiology. Girculation. 1996;93(5):1043-65.

19. White DW, Raven PB. Autonomic neural control of heart rate during dynamics exercise: revisited. J Physiol 2014; 592(12): 2491-2500

20. Tulppo MP, Mäkikallio TH, Seppänen T, Laukkanen RT, Huikuri HV. Vagal modulation of heart rate during exercise: effects of age and physical fitness. Am J Physiol. 1998;274(Pt 2):H424-9.

21. Gallo Júnior L, Maciel BC, Marin-Neto JA, Martins LE. Sympathetic and parasympathetic changes in heart rate control during dynamic exercise induced by endurance training in man. Braz J Med Bio Res. 1989;22(5):631-43.

22. Aronow WS, Dendinger J, Rokaw SN. Heart rate and carbon monoxide level after smoking highow-, and non-nicotine cigarettes. A study in male patients with angina pectoris. Ann Intern Med. 1971;74(5):697-702

23. Nadeau RA, James TN. Effects of nicotine on heart rate studied by direct perfusion of sinus code. Am J Physiol. 1967;212(4):911-6.

24. Mandel WJ, Laks M, Hayakawa H, Obayashi K, McCullen A. Cardiovascular effects of nicotine in the conscious dog. Modification by changes in autonomic tone. Am J Cardiol. 1973;32(7):947-55

25. Felber Dietrich D, Schwartz J, Schindler C, Gaspoz JM, Barthélémy JC, Tschopp JM, et al. Effects of passive smoking on heart rate variability, heart rate and blood pressure: an observational study. Int J Epidemiol. 2007:36(4):834-40

26. Lucini D, Bertocchi F, Malliani A, Pagani M. A controlled study of the autonomic changes produced by habitual cigarette smoking in healthy subjects. Cardiovasc Res. 1996;31(4):633-9.

27. Mendonca GV, Pereira FD, Fernhall B. Effects of cigarette smoking on cardiac autonomic function during dynamic exercise. Sports Sci. 2011 Jun;29(9):879-86

28. Alyan O, Kacmaz F, Ozdemir O, Maden O, Topaloglu S, Ozbakir C, et al. Effects of cigarette smoking on heart rate variability and plasma $\mathrm{N}$-terminal pro-B-type natriuretic peptide in healthy subjects: is there the relationship between both markers? Ann Noninvasive Electrocardiol. 2008;13(2):137-44

29. Kupari M, Virolainen J, Koskinen P, Tikkanen MJ. Short-term heart rate variability and factors modify ing the risk of coronary artery disease in a population sample. Am J Cardiol. 1993;72(12):897-903.

30. Rossi M, Marti G, Ricordi L, Fornasari G, Finardi G, Fratino P, et al. Cardiac autonomic dysfunction in obese subjects. Clin Sci (Lond). 1989;76(6):567-72.

31. Lambert GW, Straznicky NE, Lambert EA, Dixon JB, Schlaich MP. Sympathetic nervous activation in obesity and the metabolic syndrome-Causes, consequences and therapeutic implications. Pharmaco Ther. 2010 May;126(2):159-72.

32. Correa ALN , Tumelero S. Capacidade de VO2 máximo para indivíduos fumantes e não fumantes durante o exercício físico. EFDeportes. 2005;51(5):256-60 